\title{
Correction to: Measuring treatment effect on psoriatic arthritis-related domains: insights from the SPIRIT-H2H study at weeks 24 and 52
}

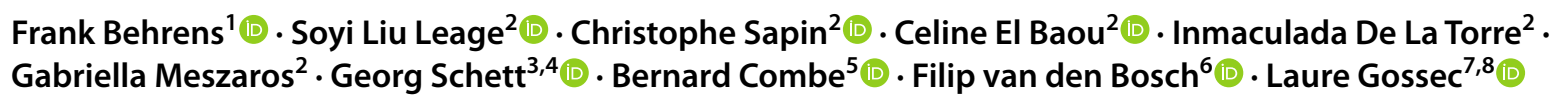

Published online: 4 November 2021

(c) The Author(s) 2021

\section{Correction to: Clinical Rheumatology}

https://doi.org/10.1007/s10067-021-05891-5

In the original published version of this article, the Figure 4 contained error. The line "ACR50 plus PASI100" has been presented incorrectly. The Figure 4 is now presented correctly.

The original article has been corrected.

Publisher's note Springer Nature remains neutral with regard to jurisdictional claims in published maps and institutional affiliations.

The online version of the original article can be found at https:// doi.org/10.1007/s10067-021-05891-5

Frank Behrens

frank.behrens@ime.fraunhofer.de

1 Dpt. of Rheumatology, Goethe University and Fraunhofer ITMP and CIMD, 60590 Frankfurt am Main, Germany

2 Eli Lilly and Company, Indianapolis, USA

3 Friedrich-Alexander-University Erlangen-Nuremberg and Universitätsklinikum, Erlangen, Germany

4 Deutsches Zentrum Für Immuntherapie, Friedrich-Alexander-University Erlangen-Nuremberg, Erlangen, Germany

5 Montpellier University, Montpellier, France

6 Ghent University Hospital, Ghent, Belgium

7 Sorbonne Université, INSERM, Institut Pierre Louis D’Epidémiologie Et de Santé Publique, Paris, France

8 Rheumatology Department, Pitié-Salpêtrière Hospital, AP-HP Sorbonne Université, Paris, France 


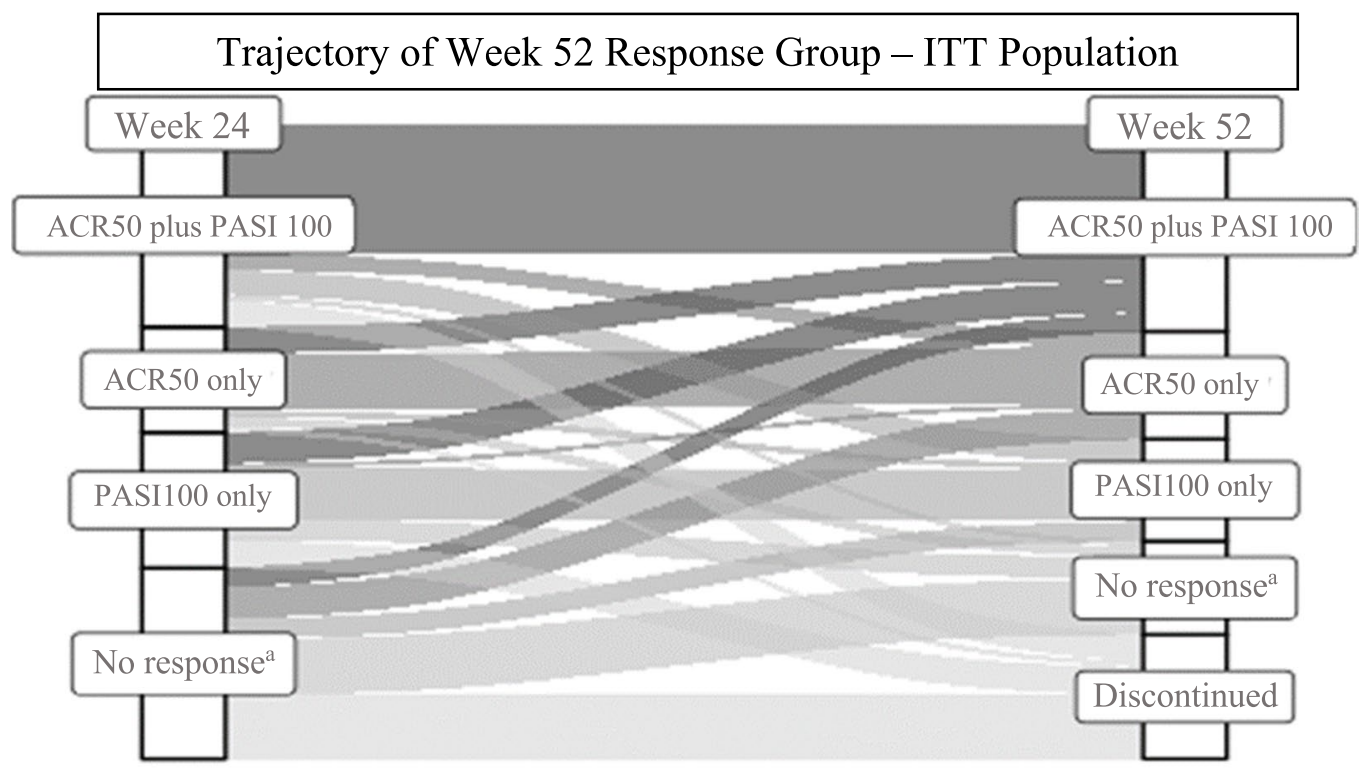

Response Group at Week 52

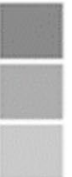

CR52 - ACR50 plus PASI100 (N=185)

JR52 - ACR50 only ( $=97)$

Not CR, JR or SR $(\mathrm{N}=83)$

Discontinued $(\mathrm{N}=87)$

NR52

SR52 - PASI100 only (N=114)

\begin{tabular}{|lccccc|}
\hline & \multicolumn{5}{c|}{ Response group at week 52, n (\%) $^{\mathbf{b}}$} \\
& $\begin{array}{c}\text { CR52 } \\
\text { (ACR50 plus } \\
\text { PASI100) } \\
\text { (N=185) }\end{array}$ & $\begin{array}{c}\text { JR52 } \\
\text { (ACR50 only) } \\
\text { (N=97) }\end{array}$ & $\begin{array}{c}\text { SR52 } \\
\text { (PASI100 } \\
\text { only) }\end{array}$ & $\begin{array}{c}\text { Neither ACR50 } \\
\text { nor PASI 100 } \\
\text { (N=83) }\end{array}$ & $\begin{array}{c}\text { Discontinued } \\
\text { (N=87) }\end{array}$ \\
ACR50 plus PASI100 & $116(62.7)$ & $14(14.4)$ & $29(25.4)$ & $5(6.0)$ & $17(19.5)$ \\
ACR50 only & $24(13.0)$ & $50(51.5)$ & $6(5.3)$ & $9(10.8)$ & $5(5.7)$ \\
PASI100 only & $28(15.1)$ & $4(4.1)$ & $57(50.0)$ & $19(22.9)$ & $13(14.9)$ \\
Neither ACR50 nor & $17(9.2)$ & $29(29.9)$ & $22(19.3)$ & $50(60.2)$ & $52(59.8)$ \\
PASI100 & & & & & \\
\hline
\end{tabular}

${ }^{a}$ Neither ACR50 nor PASI100. ${ }^{b}$ The Sankey diagram and tabulated patient numbers show the numbers and proportions of each week 52 response group who had the response specified on the left at week 24 . The four response groups were Combined Responder (CR52): patients who achieved simultaneous ACR50 and PASI100 response, Joint Responder (JR52): patients who achieved ACR50 but not PASI100 response, Skin Responder (SR52): patients who achieved PASI100 but not ACR50 response, and Nonresponder (NR52): patients who did not achieve ACR50 or PASI100 response after 52 weeks of treatment. ACR50: improvement of $\geq 50 \%$ in American College of Rheumatology criteria; CR52: combined responder at week 52; JR52: joint responder at week 52; ITT: intention to treat; NR52: nonresponder at week 52; PASI100: 100\% improvement in Psoriasis Area Severity Index score; SR52: skin responder at week 52

Fig. 4 Sankey diagram showing trajectory of response based on response group at week 52 